\title{
Editorial
}

\section{Nanomaterials for Nanooptoelectronics Device Applications}

\author{
Chien-Jung Huang, ${ }^{1}$ Chi-Chih Liao, ${ }^{2}$ Mau-Phon Houng, ${ }^{3}$ \\ Fu-Ken Liu, ${ }^{4}$ and Ying-Chung $\mathrm{Chen}^{5}$ \\ ${ }^{1}$ Department of Applied Physics, National University of Kaohsiung, Kaohsiung 81148, Taiwan \\ ${ }^{2}$ Applied Optoelectronics, Inc., Sugar Land, TX 77478, USA \\ ${ }^{3}$ Institute of Microelectronics and Department of Electrical Engineering, National Cheng Kung University, Tainan 70101, Taiwan \\ ${ }^{4}$ Department of Applied Chemistry, National University of Kaohsiung, Kaohsiung 81148, Taiwan \\ ${ }^{5}$ Department of Electrical Engineering, National Sun Yat-Sen University, Kaohsiung 80424, Taiwan
}

Correspondence should be addressed to Chien-Jung Huang; chien@nuk.edu.tw

Received 6 November 2014; Accepted 6 November 2014; Published 30 December 2014

Copyright (c) 2014 Chien-Jung Huang et al. This is an open access article distributed under the Creative Commons Attribution License, which permits unrestricted use, distribution, and reproduction in any medium, provided the original work is properly cited.

Nanomaterials are a new class of materials, with dimensions in the $0.1 \mu \mathrm{m} \sim 0.1 \mathrm{~nm}$ range, that provide great potentials of improving and enhancing the performance and functionality of many industrial products. Nanostructures, based on their shapes, can be categorized into uniform zero-dimensional (0D), elongated one-dimensional (1D), and planar twodimensional (2D) structures. The recent emphasis on the nanomaterial research is put on $1 \mathrm{D}$ nanostructures at the expense of $0 \mathrm{D}$ and $2 \mathrm{D}$ ones, perhaps due to the intriguing possibility of using them in a majority of short-term future applications. The most successful examples are seen in the microelectronic, green energy or display where these have always meant a greater performance ever since the invention of transistors, invertors, and lightings, for example, higher density of integration, faster response, lower cost, and less power consumption.

In recent years, the nanomaterials for nanooptoelectronics device applications have been highly developed in various fields, due to their flexibility and light weight for daily use. As a result, the field of nanooptoelectronics device has been the subject of intensive researches and investigations. In addition, nanooptoelectronics devices are environmentally sustainable due to the abundant availability of the nanostructured raw materials. The chance to share and discuss these crucial nanooptoelectronics developments in a timely and influential forum is important. This special issue selects 20 papers about display, solar cell materials, devices, and processing, nanoscale luminescent materials, and other related fields. This special issue enables interdisciplinary collaboration between engineering technologists and science in the industrials and academic field.

Chien-Jung Huang Chi-Chih Liao

Mau-Phon Houng

Fu-Ken Liu

Ying-Chung Chen 

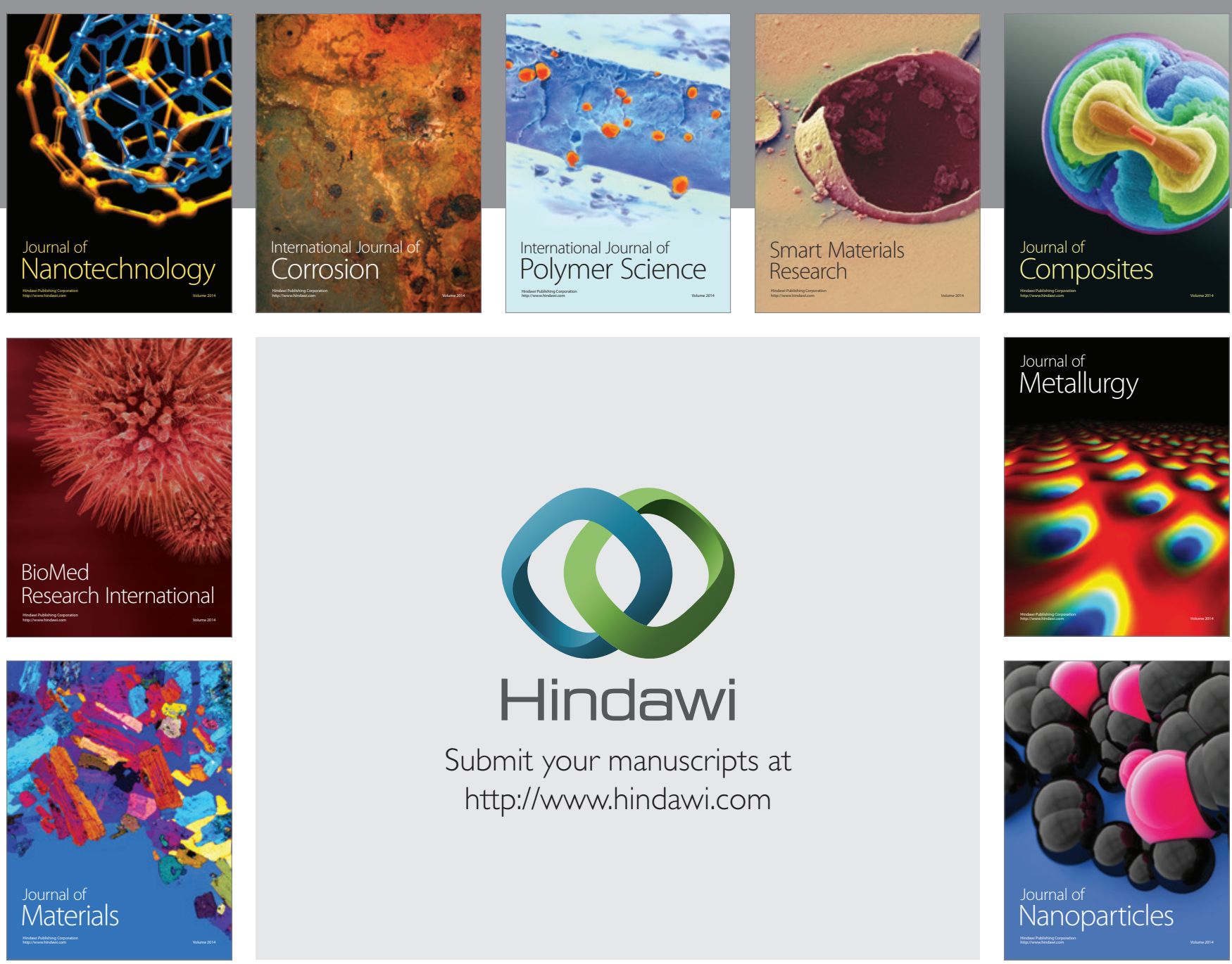

Submit your manuscripts at http://www.hindawi.com
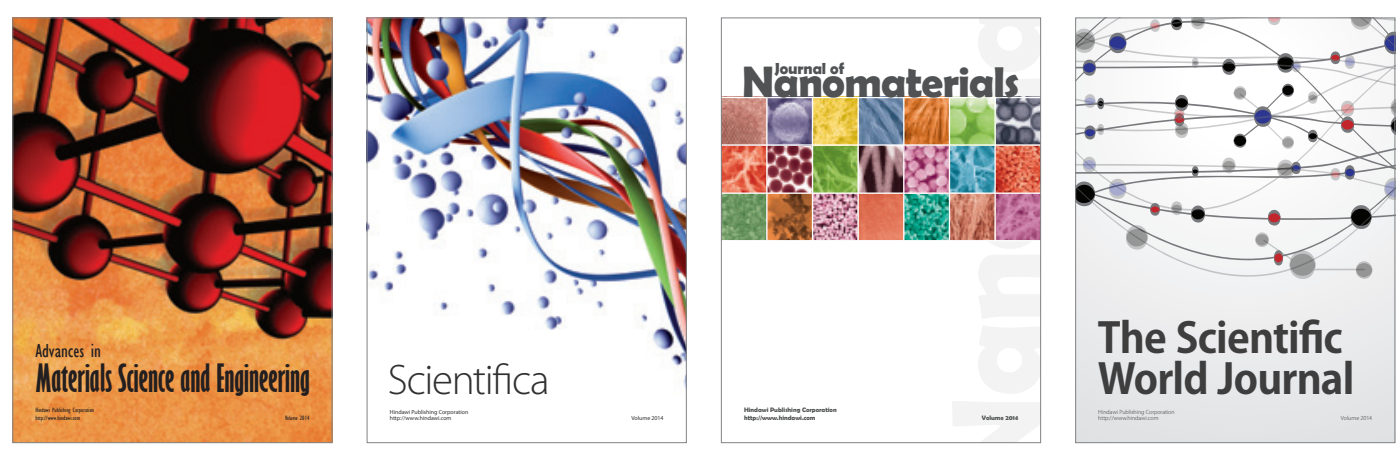

\section{The Scientific World Journal}
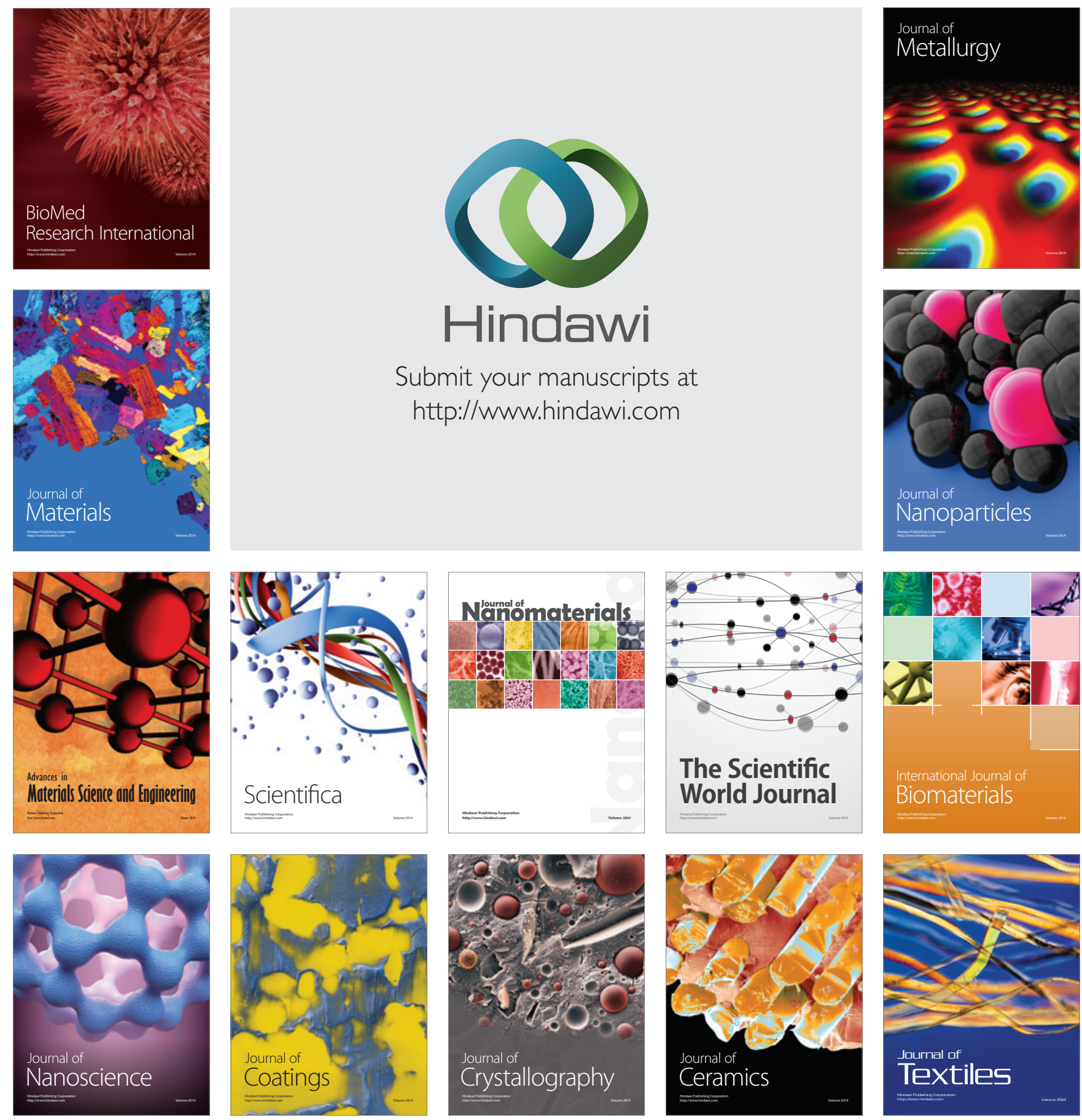